\title{
Irregular S-cone mosaics in felid retinas. Spatial interaction with axonless horizontal cells, revealed by cross correlation
}

\author{
Peter Kurt Ahnelt \\ Institut für Allgemeine und Vergleichende Physiologie, Universität Wien, Wien, Austria \\ Eduardo Fernández, Oscar Martinez, and Jose Angel Bolea \\ Bioengineering Institute, Universidad Miguel Hernández, Alicante, Spain
}

Anna Kübber-Heiss

Institut für Pathologie und Gerichtsmedizin, Veterinärmedizinische Universität, Wien, Austria

\begin{abstract}
Received September 8, 1999; revised manuscript received November 23, 1999; accepted November 24, 1999
In most mammals short-wavelength-sensitive (S) cones are arranged in irregular patterns with widely variable intercell distances. Consequently, mosaics of connected interneurons either may show some type of correlation to photoreceptor placement or may establish an independent lattice with compensatory dendritic organization. Since axonless horizontal cells (A-HC's) are supposed to direct all dendrites to overlying cones, we studied their spatial interaction with chromatic cone subclasses. In the cheetah, the bobcat, and the leopard, anti-S-opsin antibodies have consistently colabeled the A-HC's in addition to the S cones. We investigated the interaction between the two cell mosaics, using autocorrelation and cross-correlation procedures, including a Voronoi-based density probe. Comparisons with simulations of random mosaics show significantly lower densities of S cones above the cell bodies and primary dendrites of A-HC's. The pattern results in different longwavelength-sensitive-L- and S-cone ratios in the central versus the peripheral zones of A-HC dendritic fields. The existence of a related pattern at the synaptic level and its potential significance for color processing may be investigated in further studies. (C) 2000 Optical Society of America [S0740-3232(00)02203-1]
\end{abstract}

OCIS codes: $330.7310,330.1720,330.5310,330.6180,330.6130,330.5000$.

\section{INTRODUCTION}

It is generally assumed that variations of retinal photoreceptor mosaics found among species with differing lifestyles reflect the design of the eye's optics and neuronal grain. ${ }^{1}$ Basically, the retinal sensory layer serves as a testing ground for evolution.

\section{A. Mammalian S-Cone Mosaics: Generally Irregular} The possibilities of identifying short-wavelength-sensitive (S) cones in primates ${ }^{2-5}$ have initiated studies of their S-cone mosaic properties and of the consequences for spatial and chromatic processing. ${ }^{6,7}$ The fact that in most diurnal species $\mathrm{S}$ cones constitute only a minor subpopulation has been attributed to the defocusing effects of $\mathrm{S}$ image components. Application of antiopsin antibody to other mammalian species provides a more balanced view on S-cone mosaics. Irregular rather than regular patterns appears to be the rule among most mammals with S-cone mosaics (Fig. 1). This group includes marsupials such as the opossum, ${ }^{8}$ as well as rabbits, cats, horses, rats, and guinea pigs, ${ }^{2,9-11}$ but the irregularity has been also found in Tarsius, a nocturnal prosimian, ${ }^{12}$ and in both the dichromatic and the trichromatic marmoset (Callithrix), a diurnal platyrrhine monkey. ${ }^{13}$ Although not crystalline, the peripheral S-cone mosaic of humans and Old World monkeys is clearly nonrandom. ${ }^{5,14,15}$ S-cone submosaics of similar regularity have also evolved in two other mammalian groups. In both groups, the ground squirrels and their relatives ${ }^{16,17}$ and the tree shrews, ${ }^{18}$ the regular S-cone mosaics are associated with cone-dominant retinas.

\section{B. S-Cone Patterns and Connectivity to Interneurons} Over the years it has been established that most placental mammals have two types of horizontal cell (HC). ${ }^{19,20}$ The specific role of these cell types' dendritic trees, both connecting to cones, ${ }^{21,22}$ has remained an enigma. For primate HC's, it has been shown ${ }^{23-26}$ that a weighting of connectivity exists with respect to the two major classes of cones. H2-HC's (possibly a modified axonless type ${ }^{20}$ ) have elevated connection densities to $\mathrm{S}$ cones within their dendritic field but also connect to long-wavelengthsensitive (L) and middle-wavelength-sensitive (M) cones. For other mammalian species, no conclusive evidence for the existence of chromatic connection patterns has been reported.

An important approach toward probing for mutual interdependence of mosaics such as S cones and HC's is cross correlation. ${ }^{27,28}$ Recently, the method of autocorrelation by establishment of a density recovery profile has 


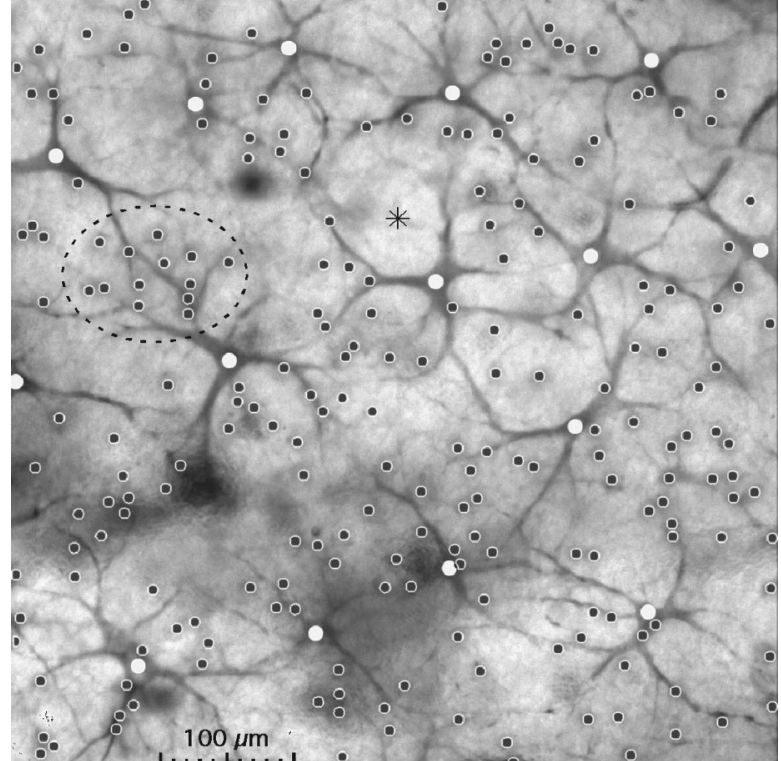

Fig. 1. Cheetah retina, superior periphery: JH455 labeling. S-cone position plotted on composite image of horizontal cell (HC) level merged from focused portions within a series of optical sections. S cones (dark spots) are irregularly arranged. Clustering preferentially appears above areas in which peripheral dendrites from HC's overlap (enclosed within dashed circle). A-HC bodies (white circle indicates nucleus position) and primary dendrites remain largely free of S cones as well as zones with few or no dendrites (asterisk).

been extended to correlate the positions of $\mathrm{S}$ cones with the positions of underlying S-cone specific bipolar cells (blue bipolars) in the primate retina. ${ }^{29}$ The result suggests that there is direct dependence between the two mosaics, whereby the blue bipolar cell positions have more degrees of freedom by virtue of the lateral range of their dendrites. Extending this concept to interneuron types with even larger dendritic range and considerable overlap such as axonless horizontal cells (A-HC's ${ }^{30,31}$ ) would imply that the two mosaics should become practically independent. If there is still correlation, it may then point to other constraints.

The present paper takes a new approach, based on the individual shapes of Voronoi domains, to probe for specific spatial correlations between HC's and spectral cone types in nonprimate mammals. It discusses possible approaches toward verification of the properties of irregular mosaics.

\section{MATERIALS AND METHODS}

\section{A. Specimens and Data Acquisition}

Eyes from three cheetahs, two European lynxes, one leopard, and two mandrills (zoo animals delivered to veterinary pathology for autopsy) were removed $20 \mathrm{~min}$ to $20 \mathrm{~h}$ after death and were fixed in $4 \%$ paraformaldehyde in phosphate buffered saline solution. Isolated retinas were prepared as whole mounts and were then labeled with one or two antibodies [we used peanut agglutinin, antiopsin JH492, and antiopsin JH455 (the last two were kindly provided by J. Nathans)], as described elsewhere. ${ }^{8}$ JH455 labeled not only the S-cone outer segments but also occasionally the complete cones, and, more importantly, it consistently led to colabeling of cell bodies and major dendrites of A-HC's (Fig. 1).

Positions of cells were mapped with an on-line videomicroscopy system (Hamamatsu 2400 camera; Apple Power Mac with Video-Monitor-Genlock software). Throughfocus series were captured digitally (Photometrix) from outer segments to HC's to create Adobe Photoshop multilayer files. Point pattern graphs were created in CANVAS or Photoshop overlay planes. For pattern analysis, $x$ and $y$ coordinates were identified with $\mathrm{NIH}$ Image software.

\section{B. Data Analysis}

The spatial organization of the S-cone and the A-HC mosaics was analyzed with the Spatial Point Pattern software $^{32}$ by means of the distribution of nearestneighbor distances..$^{33}$ Furthermore, we also used analysis of cross correlation between different arrays. ${ }^{27-29,34,35}$ We introduce what is, to our knowledge, a new procedure that is based on the Voronoi (or Dirichlet) tessellation to adapt to the frequently noncircular dendritic fields (DF's) of many A-HC's.

The diagram obtained by the method is illustrated in Fig. 2. First, a population is chosen as a reference (in our case, the A-HC's). Then the Voronoi tessellation for these cells is computed..$^{36-38}$ Each Voronoi polygon consists of all the points in the plane that are closer to one

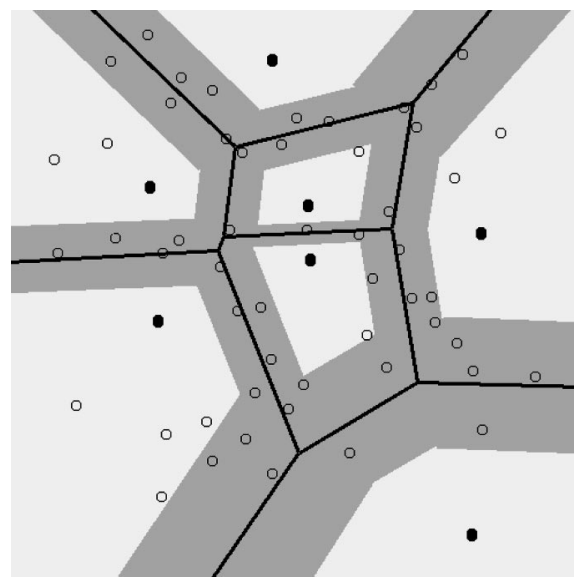

Fig. 2. Diagram obtained by the Voronoi-based cross-correlation procedure. Each Voronoi domain defines the area in the plane of the A-HC population that is closer to one A-HC than to any other A-HC of the array. The areas are uniform, indicating that A-HC's tile the retina evenly. The next step is the construction of bands around the edges of the Voronoi polygons and the computation of the number of points belonging to the upper population (S cones) inside these bands. The basic idea is that, if both populations are independent, then the occurrence of an A-HC should not alter the probability of the occurrence of $\mathrm{S}$ cones, and the average density of $\mathrm{S}$ cones should be the same inside and outside the bands. The width of each band is calculated specifically for each point and each edge of the Voronoi diagram (see Section 2 ). In this illustration the gray bands represent a bandwidth of 0.29 ( $=29 \%$ to the distance between a given A-HC and its closer Voronoi edges). For this bandwidth, the areas inside (white) and outside (gray) the polygons are equivalent, so the same number of S cones is expected ( $V$ proportion is equal to 0.5 ) inside and outside the bands. Since there are more $\mathrm{S}$ cones inside the bands, the $V$ proportion $(=0.82)$ is higher than expected and indicates a negative correlation between both mosaics. 
Table 1. Nearest-Neighbor Histograms of Cones Shown in Fig. 3

\begin{tabular}{lrcrrr}
\hline \multicolumn{1}{c}{ Cell Type } & \multicolumn{1}{c}{$n$} & Mdist $^{a}(\mu \mathrm{m})$ & $\mathrm{SD}$ & \multicolumn{2}{c}{$P^{b}$} \\
\hline Cheetah A-HC $^{2}$ & 38 & 112.7 & 23.7 & $R^{c}:$ & 0.000 \\
Cheetah SC $^{d}$ & 353 & 25.7 & 11.9 & $I^{e}:$ & 0.037 \\
Mandrill SC & 244 & 37.9 & 8.5 & $R:$ & 0.000 \\
Mandrill LC $^{f}$ & 2074 & 14.2 & 1.9 & $R:$ & 0.000 \\
\hline
\end{tabular}

${ }^{a}$ Mdist, nearest-neighbor distance.

${ }^{b} P$, probability value found by the Clark-Evans method. ${ }^{41}$

${ }^{c} R$, regular distribution.

${ }^{d}$ SC, short-wavelength-sensitive cone.

${ }^{e} I$, irregular distribution.

${ }^{f}$ LC, long-wavelength-sensitive cone.

cell than to any other cell in the mosaic. The next step is the construction of bands around the edges of the Voronoi polygons and the computation of the number of points belonging to the distal population ( $\mathrm{S}$ cones) inside these bands. The basic idea is to study the pattern of interdependence among the two component patterns. Thus, if both populations are independent, then the occurrence of a reference cell should not alter the probability of the occurrence of the sample cells, and the average density of sample cells should be the same inside and outside the bands. However, it could happen that the sample cells (e.g., S cones) show either a positive or a negative correlation with the reference cells (e.g., A-HC's). In a twodimensional array this may simply reflect the physical size of the cells; in layered samples it may be a manifestation of more-subtle effects. If reference cells inhibit sample cells, then the latter will be near the edges of the Voronoi diagram, and the number of sample cells inside the bands will be significantly higher than expected. If the number of sample cells inside the bands is decreased, it means that reference cells are positively correlated with them.

The expected proportion of cells inside the bands, provided that both populations are independent, is easily calculated. Let $\Delta r$ be the width of each band (it is not fixed for the entire area being studied but is proportional to the distance between a given point and its closer Voronoi edges, being $0<\Delta r<1$ ). Thus $\Delta r$ could be $10 \%$ of the distance from the edge to the point $(\Delta r=0.1), 20 \%$ of the distance from the edge to the point $(\Delta r=0.2)$, etc.

$\left|V\left(p_{i}\right)\right|$, area of the Voronoi polygon;

$N_{V\left(p_{i}\right)}$, number of points in $\left|V\left(p_{i}\right)\right|$;

$\left|V\left(p_{i}, \Delta r\right)\right|$, area of the Voronoi region after reduction of the Voronoi domain by a factor of $\Delta r$ (white region in Fig. 2);

$\left|V\left(p_{i}, B\right)\right|=\left|V\left(p_{i}\right)-\right| V\left(p_{i}, \Delta r\right) \mid$ (shaded region in Fig. 2);

$N_{V\left(p_{i}, \Delta r\right)}$, number of points in $\left|V\left(p_{i}, \Delta r\right)\right|$;

$N_{V\left(p_{i}, B\right)}$, number of points in $\left|V\left(p_{i}, B\right)\right|$. Then the proportion between $\left|V\left(p_{i}\right)\right|$ and $\left|V\left(p_{i}, \Delta r\right)\right|$ in a closed Voronoi polygon for every $\Delta r$ is

$$
\begin{aligned}
\left|V\left(p_{i}, \Delta r\right)\right| & =(1-\Delta r)^{2}\left|V\left(p_{i}\right)\right|, \\
\left|V\left(p_{i}, B\right)\right| & =\left|V\left(p_{i}\right)\right|\left[1-(1-\Delta r)^{2}\right] .
\end{aligned}
$$

Assuming a random distribution of points, the expected number of points inside the bands is related to the band- width; hence that number of points inside the bands $\left[N_{V\left(p_{i}, B\right)}\right]$ for a determined $\Delta r$ is

$$
N_{V\left(p_{i}, B\right)}=N_{V\left(p_{i}\right)}\left[1-(1-\Delta r)^{2}\right],
$$

and the $V$ proportion between the number of points inside the bands and the total number of points is written as

$$
N_{V\left(p_{i}, B\right)} / N_{V\left(p_{i}\right)}=1-(1-\Delta r)^{2} .
$$

This $V$ proportion varies depending on $\Delta r$, as is shown in Table 2 below. If the measured $V$ proportion is higher than expected, it means that there are more points inside the bands, which suggests a negative correlation. If the measured $V$ proportion is smaller than expected, it indicates a positive correlation.

Voronoi domains and nearest-neighbor distances were computed by use of the incremental algorithm first described in Ref. 36. To avoid the consequences of edge effects we considered only those polygons inside the convex hull of the reference points. A specific program was developed in $\mathrm{C}++$ and was compiled in a Linux station to permit performance of all the statistical and graphical techniques. The program is available on request. ${ }^{39}$ To test the significance of this measure we calculated the $V$ proportion for several $\Delta r$ values. Furthermore, we also used a Monte Carlo test procedure. This involves generating a set of two random and independent patterns, each with the same number of points as the empirical reference and upper points, in a study area identical to that of the real pattern. We did this 100 times for each field, and then the mean and the standard deviation (SD) of the $V$ proportion for each $\Delta r$ were calculated.

\section{Computer Simulations}

To simulate a negative correlation process, random $x$ - and $y$-coordinate values were generated independently for every reference and upper point by use of a Strauss-type simple inhibition process. ${ }^{37}$ Distributions were randomly generated by positioning of one cell at a time in the sampling field (identical to that of the empirical pattern) and by determination of its distance to its closest neighbor. Whenever the cell's nearest-neighbor distance was smaller than the value of the effective radius, which was determined by use of the density recovery profile on the real sample, ${ }^{27}$ the cell was eliminated. The minimum distance allowed between reference and upper points was given by the effective radius of the cross correlogram of the real sample. ${ }^{27,29}$ This process was continued until the simulated distribution reached the same cell density as that of the real mosaics. To test the statistical significance of this model's fit of the data, we used the refined nearest-neighbor analysis. ${ }^{32,37,40}$ (See Table 1 and Fig. 3.)

\section{RESULTS}

A. Histology and General Topography

Application of JH455 antibody results in consistent and intense labeling of outer segments in a minor population of cones in mammalian retinas. In the cheetah, S-cone proportions varied from $8 \%$ to $15 \%$, with a maximum of $\sim 7000 / \mathrm{mm}^{2}$ in the temporal retina and $\sim 1000 / \mathrm{mm}^{2}$ in the 
(a)

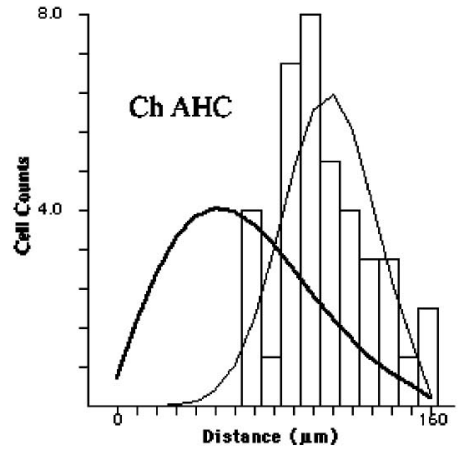

(c)

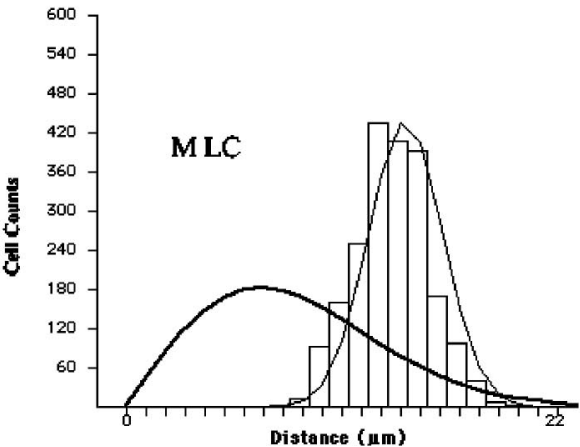

(e) (b)

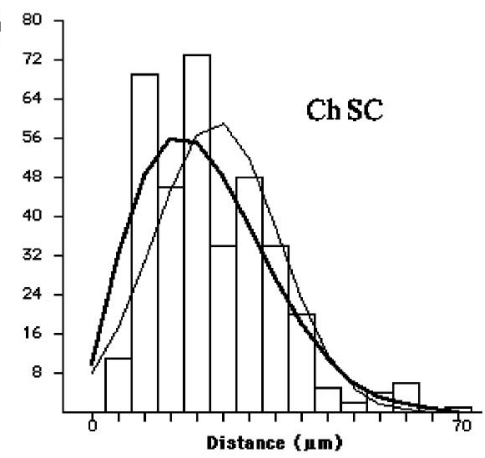

(d)

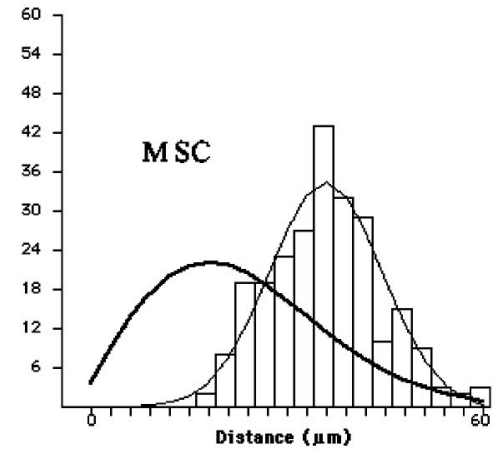

Fig. 3. Nearest-neighbor analysis of cheetah [(a), (b)] and mandrill [(c)-(e)] cell mosaics (see Table 1 for data). (a) Cheetah A-HC's (Ch AHC) show a near-Gaussian (regular) histogram. (b) The histogram of cheetah S cones (Ch SC) is close to a Rayleigh distribution, while (c) mandrill L and M cones (M LC) and (d) mandrill S cones (M SC) have regular patterns. (e) Sample of peripheral cone mosaic from mandrill retina (black, S cones; gray, putative L and M cones). Scale bar, $50 \mu \mathrm{m}$.

far periphery. At any location the mosaic of $\mathrm{S}$ cones appeared irregular [Fig. 3(b)], leading to local clustering as well as to major areas lacking labeled S cones (Fig. 1). This is in contrast to samples of $\mathrm{L}$ and $\mathrm{M}$ cones [Fig. 3(c)] and of $\mathrm{S}$ cones [Fig. 3(d)] in the peripheral retina of the mandrill, which-at different scales-have regular (Gaussian) intercone distributions. Local mosaic properties of lynx and leopard retinas were qualitatively similar to the findings in the cheetah retina. The following data refer to samples from cheetah retinas unless otherwise noted.

Occasionally, complete cones were labeled, allowing identification of pedicles linking to first-order interneurons [Fig. 4(b)]. In addition to the S-cone population, a plexus of cells having the typical characteristics of felid A-HC's ${ }^{42}$ was labeled. When judged from the position of their cell bodies, the A-HC's are regularly spaced [Fig. 3(a)], except for a few occasions in which single cells appear to be missing within the plexus. In contrast to the smooth concentric DF's of short-axon HC's, the DF outline of A-HC's is often polygonal or extremely elongated. A few major dendrites originate at irregular angles (Fig. 1) from the cell bodies, which are often ill defined and situated between their broad bases. Thus the spatial tiling derived from the cell bodies often represents a more regular pattern than is found in the patterns at the level of their eccentric dendrites. Labeling of A-HC elements was most intense and extensive close to the ora serrata. It remained visible across approximately two thirds of the retina but faded toward the central areas, presumably because the reagents did not diffuse completely where the retina is thicker.

Overlays of mapped cones [Fig. 1] onto the HC plexus allow direct inspection of possible spatial interactions. Sometimes clustering of cones is associated with sites in which only peripheral dendrites of A-HC's overlap (Fig. 4). Conversely, in areas above A-HC bodies and primary dendrites, few or no $\mathrm{S}$ cones are situated at the photoreceptor level (Fig. 1, asterisk), but these observations require specific statistical analysis. 


\section{B. Statistical Analysis of S-Cone A-HC Mosaic Interactions}

Assignment of individual S cones to $\mathrm{HC}$ 's dendrites is hardly possible solely by comparison of the overlays. Nonetheless, it may be assumed that unspecific connections to underlying mosaics will result, on average, in either regular or random distribution. Specific interactions, however, may be revealed by cross-correlation procedures.

In a first run we mapped the major part of a meridian in 18 overlapping samples and applied circular density recovery procedures. Although our results showed the
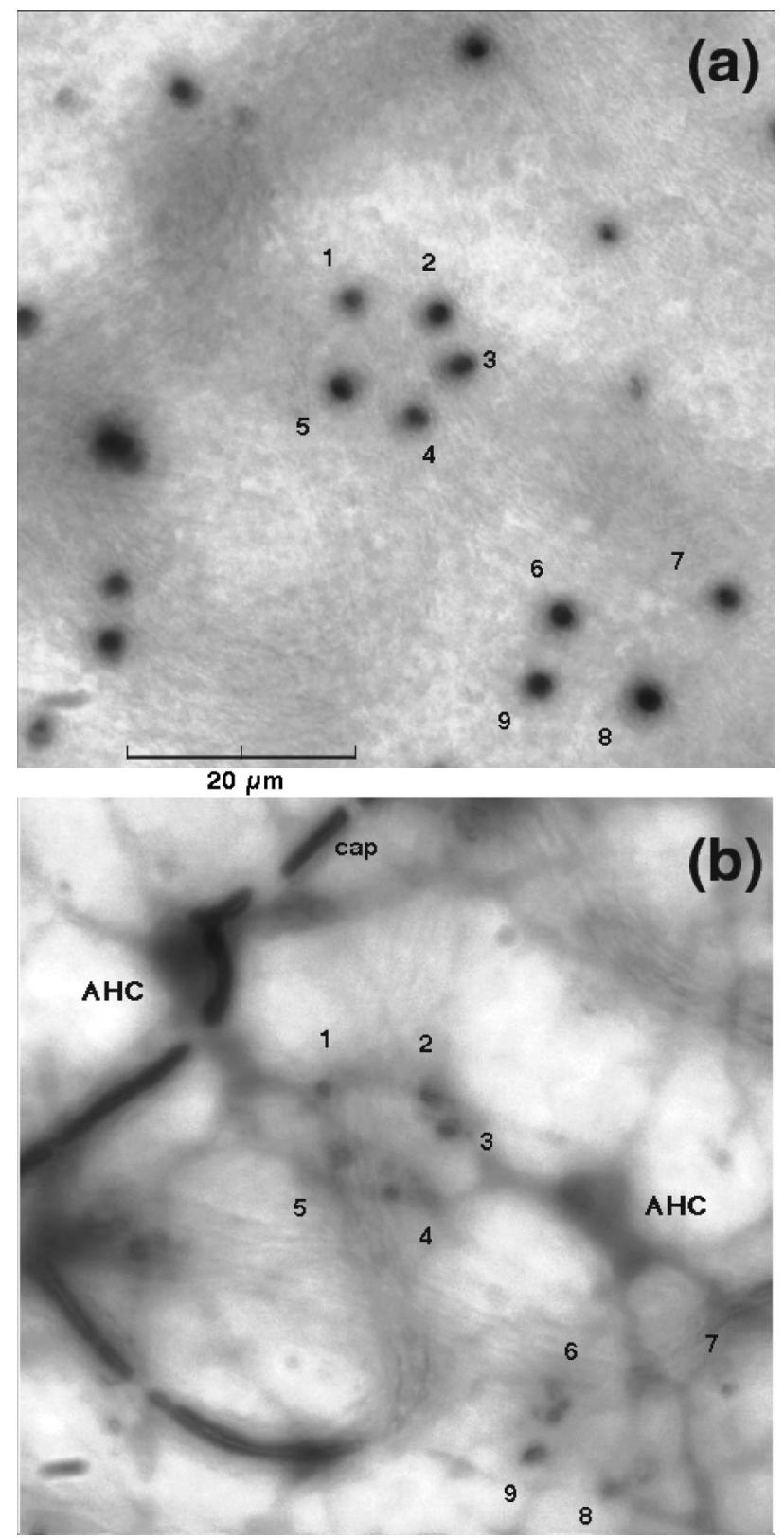

Fig. 4. Leopard retina: JH455 labeling. (a) Composite of focused portions at the level of the inner outer segment. Two clusters of S cones are numbered (1-5, 6-9). (b) Same location at the level of the outer plexiform layer. Labeled pedicles of $\mathrm{S}$ cones are located above overlapping dendrites from two HC's (AHC's). A third HC is located beyond the lower right-hand corner. Peroxidase reaction also labels erythrocytes in two capillaries (cap).

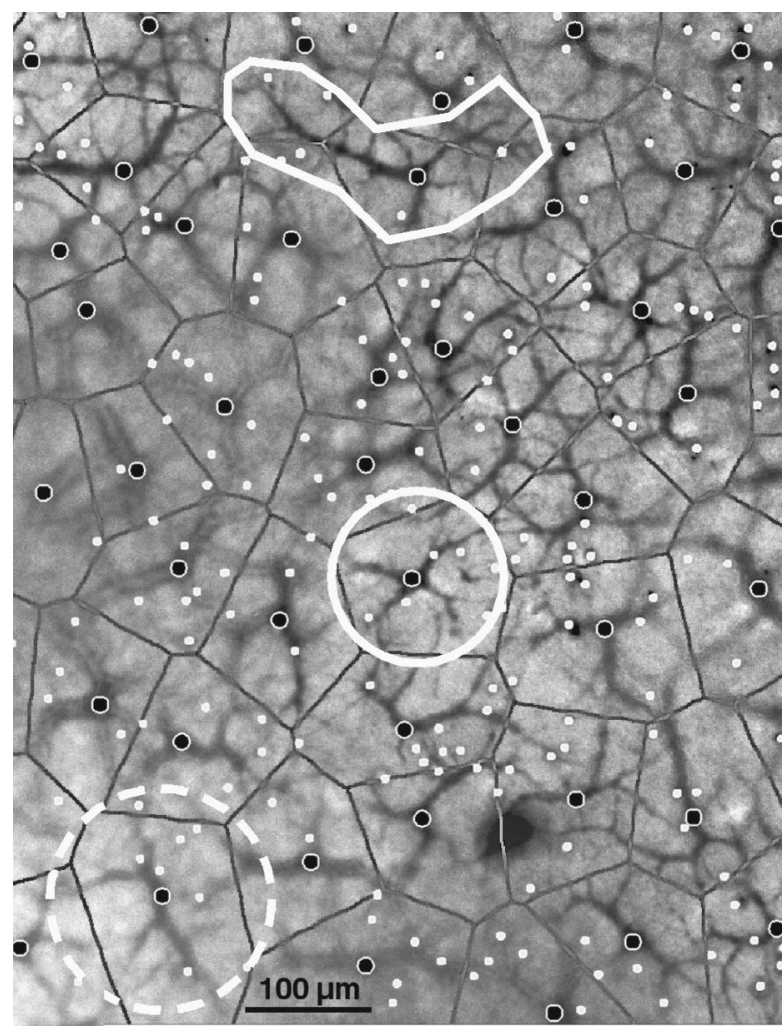

Fig. 5. Lynx retina: circular domains versus Voronoi domains. Comparison of actual A-HC branching with overlays, indicating that only in some cases are circular samplings (solid white circle) adequate approximations. Voronoi domains (polygonal grid) generally provide more-adequate spatial approximations of DF's than do circles (dashed white circle). However, since the Voronoi procedure is based solely on the locations of cell bodies and does not produce concave corners, it may also fail for cells with extreme (bipolar or angular) dendritic domains (white polygon).

presence of a minimum above $\mathrm{A}-\mathrm{HC}$ centers, we found that this cross-correlation method is not well suited for noncircular fields. The shape of Voronoi domains is derived from individual intercell distances and therefore may represent a better representation of DF's. Thus we designed a two-step tessellation procedure to produce such polygonal reference areas instead (Figs. 2 and 5, 6).

\section{Triple-Labeling Analysis}

Fluorescein isothiocyanate labeling of L-cone outer segments with JH492 reveals the population of cones [fluorescent: white spots in Fig. 6(a)], in addition to JH455DAB-labeled S cones (black spots) and A-HC's [Fig. 6(b)]. This allows one to plot comparative cross-correlation analysis for both cone subpopulations. As is evident from the mappings [Fig. 6(c)], L-cone positions are regularly spaced. Their density does not change significantly between positions above A-HC bodies versus the bands above peripheral dendrites [Fig. 7(a)]. S cones [DAB labeled: black spots in Fig. 6(d)], however, aggregate above eccentric areas [Fig. 6(d)], resulting in significant overrepresentation in Voronoi bands [Fig. 7(b), see Table 2]. Maximum deviation $(\sim 6.5 \%)$ occurs at bandwidths of $30 \%$, which covers $\sim 50 \%$ of all the Voronoi areas. 

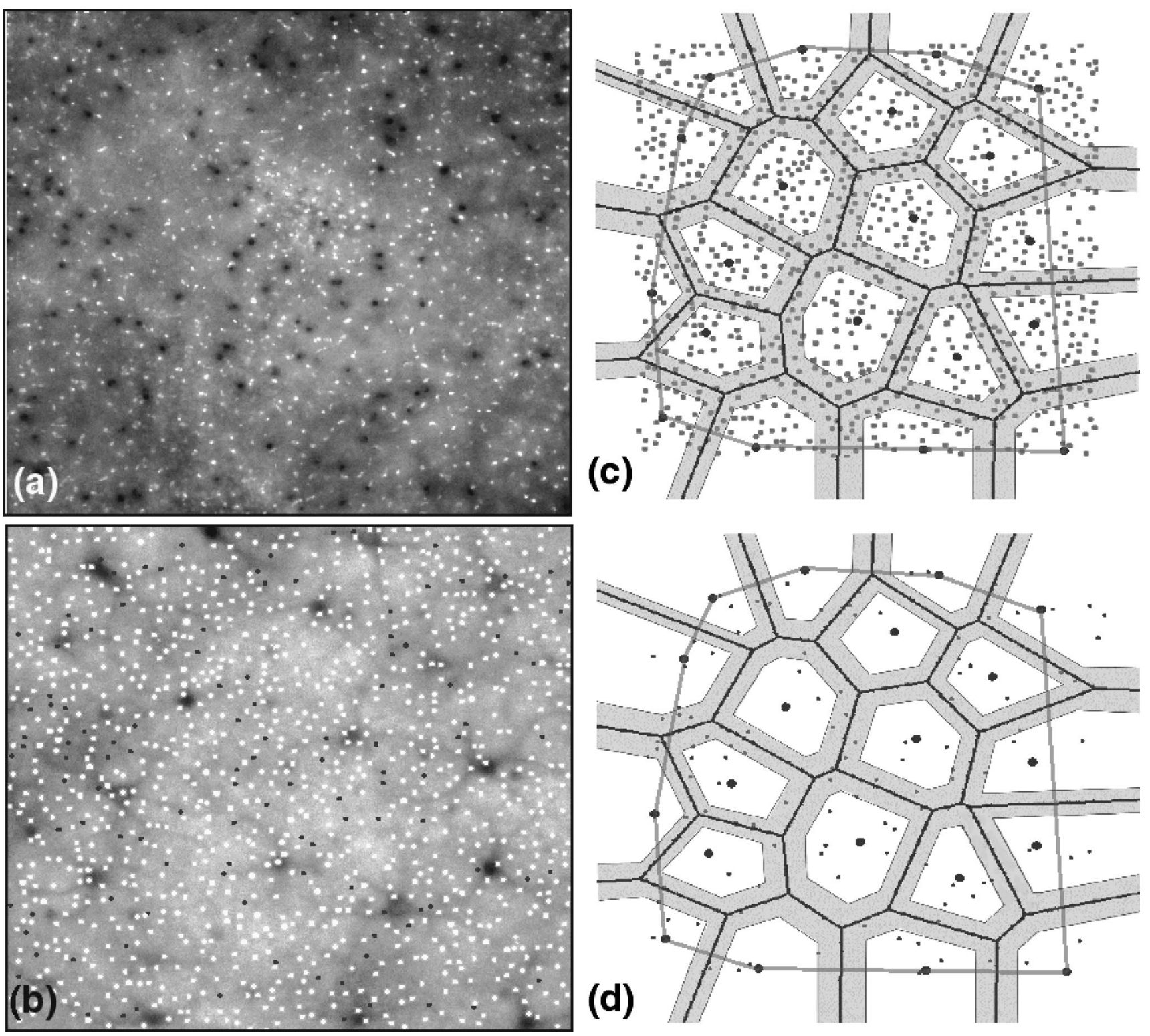

Fig. 6. Cheetah retina: double labeling with JH455 (S cones) and JH492 (L cones). (a) Minor population of S cones visualized with DAB (black spots) is interspersed between fluorescent outer segments of L cones visualized with fluorescein isothiocyanate (white spots). (b) Plane of labeled A-HC's with overlay of mapped cones. (c) Voronoi analysis of L-cone mosaic. Local L-cone density is not influenced by the underlying A-type HC organization. (d) Voronoi analysis of S-cone mosaic. S cones are preferentially located in and near the Voronoi bands. Solid lines connecting filled points in (c) and (d) demarcate measurement area (convex hull).

Table 2. Expected and Actual Percentages of $\mathrm{L}$ and $\mathrm{S}$ Cones in A-HC Voronoi Bands of Increasing Thickness ${ }^{a}$

\begin{tabular}{|c|c|c|c|c|c|}
\hline \multicolumn{3}{|c|}{ Percentage of L Cones in Band } & \multicolumn{3}{|c|}{ Percentage of S Cones in Band } \\
\hline $\begin{array}{c}\text { Bandwidth } \\
(\%)\end{array}$ & $\underset{(\%)}{\text { Simulation }}$ & L cones & $\begin{array}{c}\text { Bandwidth } \\
(\%)\end{array}$ & $\begin{array}{c}\text { Simulation } \\
(\%)\end{array}$ & S cones \\
\hline 10 & $17.90 \pm 1.79$ & 18.96 & 10 & $18.15 \pm 1.95$ & 20.86 \\
\hline 20 & $34.10 \pm 2.16$ & 35.67 & 20 & $34.09 \pm 2.57$ & 38.74 \\
\hline 30 & $48.55 \pm 2.21$ & 50.26 & 30 & $48.60 \pm 2.60$ & 55.30 \\
\hline 40 & $61.65 \pm 2.01$ & 63.13 & 40 & $61.49 \pm 2.62$ & 64.24 \\
\hline 50 & $72.73 \pm 2.19$ & 73.34 & 50 & $72.49 \pm 2.52$ & 74.83 \\
\hline
\end{tabular}

${ }^{a}$ See Fig. 7. 


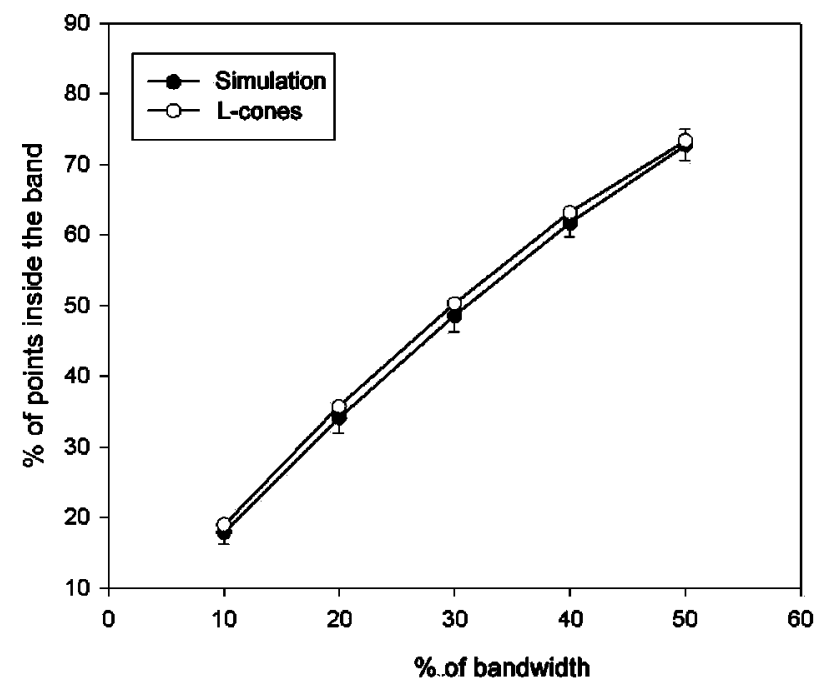

(a)

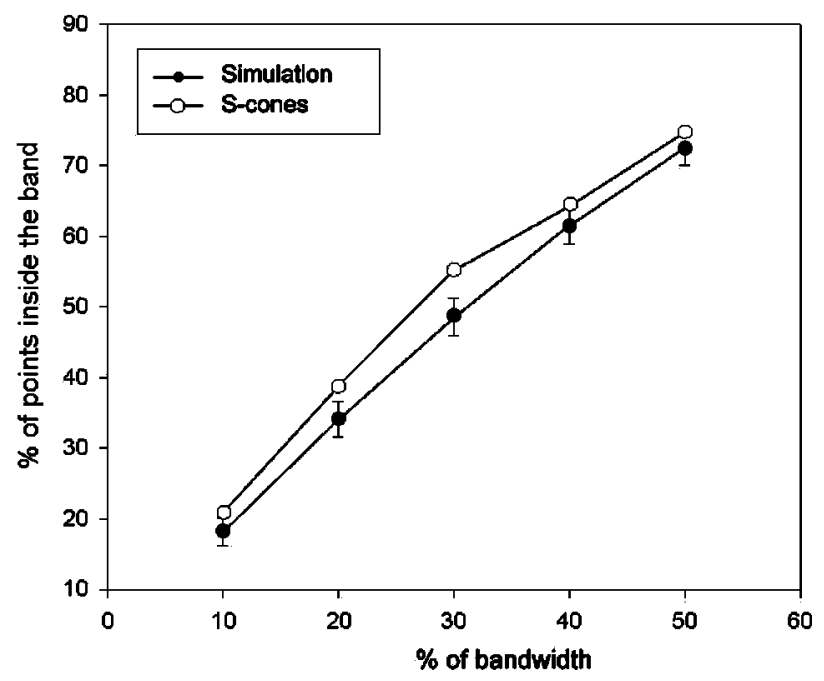

(b)

Fig. 7. Cones expected from simulation and cones actually found inside A-HC Voronoi bands from cheetah retina. (a) Plots of $\mathrm{L}$ cones and simulations. The filled circles show the results (mean \pm SD) of 100 simulated random fields with the same number of L cones located in a study area identical to that of the real pattern. The relative proportions of $\mathrm{L}$ cones do not differ from those derived from the simulation at any bandwidth (10-50\%). (b) Plot of S cones (open circles). Although the S-cone mosaic is close to random arrangement, it shows consistent and significant $(P>0.01)$ overrepresentation in bands including $10-50 \%$ of the Voronoi domain. Maximum deviation is at $30 \%$ bandwidth, equivalent to $\sim 50 \%$ portions of the Voronoi areas (Table 2 ).

\section{DISCUSSION}

The findings of the present study support the view that the seemingly random mosaic of $\mathrm{S}$ cones is in fact biased in relation to an underlying matrix of attractors or repellers coincident with the A-HC dendrites and cell bodies.

The reason for the colabeling of A-HC's by the anti-Sopsin antibody JH455 is still unclear. Labeling did not occur in the domestic cat (two trials; see also Ref. 43) and has not yet been observed in retinas of other species. This may imply that the phenomenon is limited to a few species or that it is related to our specimen acquisition procedures, which allow, for example, for better transreti- nal diffusion of antibodies. It could also be that we were labeling nonphotoreceptoral opsins ${ }^{44-46}$ or that felid HC's share some transcriptional segments with $\mathrm{S}$ cones, such as has recently been reported for bipolar cells in mice. ${ }^{47}$ In any case, the double labeling of all $\mathrm{S}$ cones and major portions of the A-HC plexus allowed us to analyze the interaction of the two mosaics by cross correlation.

\section{A. Regular versus Irregular S-Cone Mosaics}

It seems that, among mammals, regular mosaics of $\mathrm{S}$ cones are present only in some species with partially or totally cone-dominant retinas such as diurnal Old World primates, Scandentia (Tupaia) and Sciurids. These groups are likely to have evolved independently from nocturnal or crepuscular ancestors. Thus a regular S-cone mosaic may provide a particular advantage for diurnal (arboreal) habitats. The existence and the nature of such a potential benefit remain obscure and seem dispensable for most mammals, including other primates (see Subsection 1.A above).

B. Connectivity Patterns for Irregular S-Cone Mosaics It is important to emphasize that the methodology of the present study shows statistical correlation but does not provide direct evidence for related connectivity patterns of S-cone mosaics and of A-HC's. Detailed reconstructions of synaptic circuitry at the cone-HC interface and intracellular labeling of HC's, combined with S-cone labeling, are necessary to clarify the chromatic microtopography. Taking into consideration this important restriction, we may discuss implications rendered possible by this spatial interaction.

In primates the S-cone and the blue-bipolar mosaics appear to adapt to each other in both regular ${ }^{29}$ and irregular ${ }^{48}$ patterns. For equids, an A-HC connecting specifically to $\mathrm{S}$ cones has been described, ${ }^{9}$ and a similar HC type seems to be present in a marsupial. ${ }^{49}$ Both these examples suggest that, in spite of the apparent irregularity of S-cone mosaics, specific connection patterns and even cell types may exist by means of adaptive dendritic branching of interneurons. ${ }^{50}$

Comparing A-HC intercell distances with DF dimensions in the cat ${ }^{31}$ and the rabbit ${ }^{30}$ implies that each retinal location may be covered by dendrites of at least three to four A-HC's. This implies that for $(\mathrm{S})$ cones no specific spatial arrangement should be necessary to establish A-HC contacts. Still, for felid retinas, the present study demonstrates partial decomposition of the cone subtypes above A-HC DF's (Figs. 1, 6, and 7). The overall increase of the percentage of $\mathrm{S}$ cones in the peripheral bands of A-HC Voronoi domains [Fig. 6(b) and Table 2] versus the portions around the cell bodies is significant but may not seem substantial for functional consequences. Several factors, such as specific synaptic affinity and position within the DF's, may magnify the effect, as has been shown for primate HC's. ${ }^{24}$

For rabbit HC's, a recent study ${ }^{51}$ based on combining opsin antibody labeling with intracellular single-cell labeling (Procion Yellow) suggests that A-HC's connect to both L- and S-cone types. In principle, this statement does not interfere with the possibility, set forth here, of spatial segregation. Indeed, the figures presented in this 
paper suggest that a similar correlation of $\mathrm{S}$ cones and A-HC's may prevail in the dorsal retina (in the rabbit, chromatic interactions seem regionally restricted ${ }^{52}$ owing to the dorsoventral gradient of photopigment coexpresion). In addition, an electron microscopic reconstruction of a rabbit $\mathrm{A}-\mathrm{HC}^{53}$ shows connections to the majority of overlying pedicles. It was noted, however, that a few pedicles in the center of the DF were found to lack invaginating dendrites from this cell. ${ }^{53}$ In accordance with the implications of the present study, it seems possible that those pedicles are S-cone pedicles avoided by the reconstructed cell and that they are possibly connected to overlapping peripheral dendrites of neighboring A-HC's.

\section{Functional Implications}

The spatial anticlustering of $\mathrm{S}$ cones above A-HC's may have consequences for postreceptoral pathways, provided that there are related connectivity patterns. In this case the periphery of A-HC dendrites and associated bipolars would receive mixed input from both $\mathrm{S}$ and $\mathrm{L}$ cones, whereas around the soma primary dendrites and bipolars may connect mainly to $\mathrm{L}$ cones. The resolution of this chromatic landscape may be coarse but could suffice for the requirements of S-cone-related channels (lower spatial and temporal resolution). Whether this will actually lead to local inhomogeneities with respect to spatial grain and sensitivity will depend on the quality of the image itself and on the actual preservation or integration of positional information in the subsequent pathways.

\section{Developmental Aspects}

The ontogenetic events leading to such correlated mosaic patterns are still not clear. In primates ${ }^{54}$ and rats ${ }^{55}$ a set of cones-possibly with S-opsin expression-may precede further differentiation. Should relatively fixed S-cone positions be implemented first, establishing connectivity sites would require increased flexibility from elements in the underlying mosaics, such as blue bipolars. ${ }^{13,29}$

Also, S-opsin expression in a first set of cones might be influenced by establishment of primary connections with dendrites of postmitotic HC's. After further growth of the dendrites, as well as of the overall retina, these positions might end up being displaced from the $\mathrm{HC}$ body, while other cones might follow a default (green) course of expression or might be transdifferentiated. ${ }^{55}$

Such interaction could be driven by spatially restricted inhibitory factors in A-HC's during differentiation. In this sense, it is notable that different isoforms of glutamic acid decarboxylase (GAD), the enzyme that synthesizes $\gamma$-aminubutyric acid, have spatially distinct expression in rabbit HC's. ${ }^{56}$ GAD67 is expressed throughout the A-HC's and short-axon HC's, while GAD65 is restricted to A-HC somata and primary dendrites.

\section{CONCLUSION}

We have examined examples of the seemingly random organization of $\mathrm{S}$ cones that appears to be prevalent in most mammals. In the retinas of three felid species additional labeling of axonless horizontal cells (A-CH's) has enabled us to test whether the arrangement of these cells exerts a positional influence on the cone submosaics.
By the introduction of concentric Voronoi domains derived from $\mathrm{A}-\mathrm{HC}$ nuclei positions as locally adaptive elements for cross correlation, a concentrical gradient was revealed above A-HC dendritic fields for $\mathrm{S}$ but not for $\mathrm{L}$ cones. Detailed structural, developmental, and physiological analyses are necessary to clarify whether this spatial correlation also manifests itself in complementary patterns of chromatic connectivity. At present, a gap between knowledge and synthetic concepts exists, particularly for the retinal mosaics of mammals other than primates. Discovering further details of the cone-HC interfaces in these and other species will help us to achieve a more unified view of mammalian photoreceptor circuitry.

\section{ACKNOWLEDGMENTS}

Part of this work was supported by a bilateral travel grant from Austria and Spain. The authors are grateful to J. Nathans for providing the antibodies and to $\mathrm{N}$. Kouyama for supplying information on the density recovery profile procedure. C. Schubert provided expert technical assistance with specimen preparation and data acquisition.

P. Ahnelt can be reached by e-mail at peter.ahnelt@univie.ac.at.

\section{REFERENCES}

1. A. Hughes, "The topography of vision in mammals of contrasting life style: comparative optics and retinal organization," in Visual System of Vertebrates, F. Crescitelli, ed. (Springer, Berlin, 1972), Vol. 7, pp. 613-756.

2. A. Szél, T. Diamantstein, and P. Röhlich, "Identification of the blue-sensitive cones in the mammalian retina by antivisual pigment antibody," J. Comp. Neurol. 273, 593-602 (1988).

3. P. K. Ahnelt, H. Kolb, and R. Pflug, "Identification of a subtype of cone photoreceptor, likely to be blue sensitive, in the human retina," J. Comp. Neurol. 255, 18-34 (1987).

4. F. M. de Monasterio, S. J. Schein, and E. P. McCrane, "Staining of blue-sensitive cones of the macaque retina by a fluorescent dye," Science 213, 1278-1281 (1981).

5. C. A. Curcio, K. A. Allen, K. R. Sloan, C. L. Lerea, J. B. Hurley, I. B. Klock, and A. H. Milam, "Distribution and morphology of human cone photoreceptors stained with antiblue opsin," J. Comp. Neurol. 312, 610-624 (1991).

6. D. R. Williams, "Seeing through the photoreceptor mosaic," Trends Neurosci. 9, 193-198 (1986).

7. D. R. Williams and R. Collier, "Consequences of spatial sampling by a human photoreceptor mosaic," Science 221, 385-387 (1983).

8. P. K. Ahnelt, J. N. Hokoç, and P. Röhlich, "Photoreceptors in a primitive mammal, the South American opossum, $D i$ delphis marsupialis aurita: characterization with antiopsin immunolabeling," Visual Neurosci. 12, 793-804 (1995).

9. D. Sandmann, B. B. Boycott, and L. Peichl, "Blue-cone horizontal cells in the retinae of horses and other equidae," J. Neurosci. 16, 3381-3396 (1996).

10. A. Szél, P. Röhlich, A. R. Caffe, B. Juliusson, G. Aguirre, and T. van Veen, "Unique topographic separation of two spectral classes of cones in the mouse retina," J. Comp. Neurol. 325, 327-342 (1992).

11. Á. Szél and P. Röhlich, "Two cone types in rat retina detected by anti-visual pigment antibodies," Exp. Eye Res. 55, 47-52 (1992)

12. A. Hendrickson, H. Djajad, and D. Sajuthi, "The nocturnal 
simian Tarsius has short wavelength cones in an unusual topography,” Invest. Ophthalmol. Visual Sci. Suppl. 40, 238 (1999).

13. P. M. Martin and U. Grünert, "Analysis of the short wavelength-sensitive ('blue') cone mosaic in the primate retina: comparison of New World and Old World monkeys," J. Comp. Neurol. 406, 1-14 (1999)

14. M. B. Shapiro, S. J. Schein, and F. M. de Monasterio "Regularity and structure of the spatial pattern of blue cones of macaque retina," J. Am. Stat. Assoc. 80, 803-812 (1985).

15. C. A. Curcio and K. R. Sloan, "Packing geometry of human cone photoreceptors: variation with eccentricity and evidence for local anisotropy," Visual Neurosci. 9, 169-180 (1992).

16. K. O. Long and S. K. Fisher, "The distributions of photoreceptors and ganglion cells in the California ground squirrel, Spermophilus beecheyi," J. Comp. Neurol. 221, 329-340 (1983).

17. P. K. Ahnelt, "Characterization of the color related receptor mosaic in the ground squirrel retina," Vision Res. 25, 15571567 (1985)

18. B. Müller and L. Peichl, "Topography of cones and rods in the tree shrew retina," J. Comp. Neurol. 282, 581-594 (1989).

19. A. Gallego, "Horizontal cells of the Tetrapoda retina," Prog. Clin. Biol. Res. 113, 9-29 (1982).

20. L. Peichl, D. Sandmann, and B. B. Boycott, "Comparative anatomy and function of mammalian horizontal cells," in Development and Organization of the Retina, L. M. Chalupa and B. L. Finlay, eds. (Plenum, New York, 1998).

21. B. B. Boycott and H. Kolb, "The horizontal cells of the rhesus monkey retina," J. Comp. Neurol. 148, 115-139 (1973).

22. H. Kolb, A. Mariani, and A. Gallego, "A second type of horizontal cell in the monkey retina," J. Comp. Neurol. 189, 31-44 (1980)

23. P. K. Ahnelt and H. Kolb, "Horizontal cells and cone photoreceptors in primate retina: a Golgi-light microscopic study of spectral connectivity," J. Comp. Neurol. 343, 387405 (1994).

24. P. K. Ahnelt and H. Kolb, "Horizontal cells and cone photoreceptors in human retina: a Golgi-electron microscopic study of spectral connectivity," J. Comp. Neurol. 343, 406427 (1994).

25. D. M. Dacey, B. B. Lee, D. K. Stafford, J. Pokorny, and V. C. Smith, "Horizontal cells of the primate retina: cone specificity without spectral opponency," Science 271, 656-659 (1996).

26. T. L. Chan and U. Grünert, "Horizontal cell connections with short wavelength sensitive cones in the retina: a comparison between New World and Old World primates," J. Comp. Neurol. 393, 196-209 (1998).

27. R. W. Rodieck, "The density recovery profile: a method for the analysis of points in the plane applicable to retinal studies," Visual Neurosci. 6, 95-111 (1991).

28. R. W. Rodieck and D. W. Marshak, "Spatial density and distribution of choline acetyltransferase immunoreactive cells in human, macaque, and baboon retinas," J. Comp. Neurol. 321, 46-64 (1992)

29. N. Kouyama and D. W. Marshak, "The topographical relationship between two neuronal mosaics in the short wavelength-sensitive system of the primate retina," Visual Neurosci. 14, 159-167 (1997).

30. S. L. Mills and S. C. Massey, "Distribution and coverage of A- and B-type horizontal cells stained with neurobiotin in the rabbit retina," Visual Neurosci. 11, 549-560 (1994)

31. H. Wässle, L. Peichl, and B. B. Boycott, "Topography of horizontal cells in the retina of the domestic cat," Proc. R. Soc. London Ser. B. 203, 269-291 (1978).

32. E. Fernández, N. Cuenca, and J. De Juan, “A compiled BASIC program for analysis of spatial point patterns, application to retinal studies," J. Neurosci. Methods 50, 1-15 (1993).

33. H. Wässle and H. J. Riemann, "The mosaic of nerve cells in the mammalian retina," Proc. R. Soc. London Ser. B 200 , 441-461 (1978)

34. J. Cook, "Getting to grips with neuronal diversity," in Development and Organization of the Retina, L. M. Chalupa and B. L. Finlay, eds. (Plenum, New York, 1998), pp. 91120.

35. J. E. Cook, "Spatial properties of retinal mosaics: an empirical evaluation of some existing measures," Visual Neurosci. 13, 15-30 (1996).

36. P. J. Green and R. Sibson, "Computing Dirichlet tessellations in the plane," Comput. J. (Cambridge) 21, 68-173 (1978)

37. P. J. Diggle, Statistical Analysis of Spatial Point Patterns (Academic, New York, 1983).

38. L. Galli-Resta, E. Novelli, Z. Kryger, G. H. Jacobs, and B. E. Reese, "Modelling the mosaic organization of rod and cone photoreceptors with a minimal spacing rule," Eur. J. Neurosci. 11, 1461-1469 (1999).

39. Requests for this program may be sent to O. Martinez at the address given on the title page.

40. B. N. Boots and A. Getis, Point Pattern Analysis (Sage, Beverly Hills, Calif., 1988).

41. P. J. Clark and F. C. Evans, "Distance to nearest neighbor as a measure of spatial relationship in populations," Ecology 34, 445-453 (1954).

42. H. Wässle, B. B. Boycott, and L. Peichl, "Receptor contacts of horizontal cells in the retina of the domestic cat," Proc. R. Soc. London Ser. B 203, 247-267 (1978).

43. L. Peichl, Max-Planck-Institut für Hirnforschung, 60528 Frankfurt a.M., Germany (personal communication, 1999).

44. B. G. Soni and R. G. Foster, "A novel and ancient vertebrate opsin," FEBS Lett. 406, 279-283 (1998).

45. H. Sun, D. J. Gilbert, N. G. Copeland, N. A. Jenkins, and J. Nathans, "Peropsin, a novel visual pigment-like protein located in the apical microvilli of the retinal pigment epithelium," Proc. Natl. Acad. Sci. USA 94, 9893-9898 (1997).

46. I. Provencio, G. Jiang, W. J. De Grip, W. P. Hayes, and M. D. Rollag, "Melanopsin: an opsin in melanophores, brain, and eye," Proc. Natl. Acad. Sci. USA 95, 340-345 (1998)

47. M. I. Chiu and J. Nathans, "A sequence upstream of the mouse blue visual pigment gene directs blue cone-specific transgene expression in mouse retinas," Visual Neurosci. 11, 773-780 (1994)

48. X. Luo, K. K. Gosh, P. R. Martin, and U. Grünert, "Analysis of two types of cone bipolar cells in the retina of a New World monkey, the marmoset, Callithrix jacchus," Visual Neurosci. 16, 707-719 (1999).

49. S. J. N. Hokoç, M. Medeiros De Oliveira, and P. K. Ahnelt, "Three types of horizontal cells in a primitive mammal, the opossum (Didelphis marsupialis aurita)," Invest. Ophthalmol. Visual Sci. Suppl. 34, 1152 (1993).

50. E. V. Famiglietti, Jr., "Functional architecture of cone bipolar cells in mammalian retina," Vision Res. 21, 1559-1563 (1981).

51. I. Hack and L. Peichl, "Horizontal cells of the rabbit retina are non-selectively connected to the cones," Eur. J. Neurosci. 11, 2261-2274 (1999).

52. R. Pflug and H. Reitsamer, "Topographic variation of conecone interaction in spectral sensitivities determined from small field ERGs of rabbit retina," Invest. Ophthalmol. Visual Sci. Suppl. 40, 238 (1999).

53. E. Raviola and R. F. Dacheux, "Axonless horizontal cells of the rabbit retina: synaptic connections and origin of the rod aftereffect," J. Neurocytol. 19, 731-736 (1990).

54. K. C. Wikler and P. Rakic, "An array of earlydifferentiating cones precedes the emergence of the photoreceptor mosaic in the fetal retina," Proc. Natl. Acad. Sci. USA 91, 6534-6538 (1994).

55. A. Szél, B. Vigh, T. van Veen, and P. Röhlich, "Development of cone distribution patterns in mammals," in Development and Organization of the Retina, L. M. Chalupa and B. L. Finlay, eds. (Plenum, New York, 1998), pp. 43-59.

56. M. A. Johnson and N. Vardi, "Regional differences in GABA and GAD immunoreactivity in rabbit horizontal cells," Visual Neurosci. 15, 743-753 (1998). 